\title{
Short-Term Effectiveness of Medication and Psychosocial Intervention in a Cohort of Newly Diagnosed Patients With Inattention, Impulsivity, and Hyperactivity Problems \\ Journal of Attention Disorders \\ 14(2) 147-156 \\ (C) 2010 SAGE Publications \\ Reprints and permission: \\ sagepub.com/journalsPermissions.nav \\ DOI: | 0.1 | 77// $087054709347 \mid 73$ \\ http://jad.sagepub.com \\ (S)AGE
}

\author{
Bruno Falissard, ${ }^{1,2,3}$ David Coghill, ${ }^{4}$ Aribert Rothenberger, ${ }^{5}$ and \\ Maria Lorenzo ${ }^{6}$ on behalf of the ADORE Study Group
}

\begin{abstract}
Objective:The article discusses the ADHD Observational Research in Europe (ADORE) study that examined the impact of early treatment choices on outcome within the first few months, in previously untreated children with impairing inattention, impulsivity, and hyperactivity. Methods: Data are collected from a longitudinal, observational study conducted in 10 European countries that involve I,478 children (aged 6 to 18 years) with symptoms of hyperactivity, impulsivity, or inattentiveness. Linear model with a propensity score adjustment compares the decrease in ADHD-RS scores between baseline and last recorded visit across treatment groups ( 2 to 5 months). Results: At baseline, $49.9 \%$ of participants are prescribed pharmacotherapy and $44.3 \%$ a psychosocial intervention. Analysis of the effect of treatment on the evolution of ADHD-RS scores shows a positive effect of medications and either an insignificant or negative effect of psychosocial intervention. Conclusions: Early use of medication effectively reduces ADHD symptoms in routine clinical practice in Europe. The effect of psychosocial intervention has to be interpreted cautiously because the number, length, and level of standard of the sessions are not taken into account in the analyses. (J. of Att. Dis. 2007)
\end{abstract}

\section{Keywords}

ADHD, treatment evaluation, cohort study, medication, psychosocial intervention

\section{Introduction}

There is strong evidence to support the use of medication in the management of inattention, impulsivity, and hyperactivity symptoms in school-age children (McClellan \& Werry, 2003; Taylor et al., 2004). Although there is clinical trial evidence to support the use of nonpharmacological interventions, these data suggest a much smaller effect (Abikoff et al., 2005; MTA Cooperative Group, 1999) and that these treatments are more generally indicated for less pervasive and severe ADHD or as adjunctive treatments for those with coexisting mental health conditions, such as depression and anxiety (Rappley, 2005; Santosh et al., 2005; Taylor et al., 2004).

Scientific data concerning treatment evaluation in child psychiatry in general and in ADHD in particular raise several important questions (Strom, 2005). In general, treatment trials are conducted under optimal conditions that are very different from those found in daily clinical practice. They enroll carefully selected participants who are also likely to be different from those seen in routine clinical care. This can make it difficult to generalize these results and translate them into usual clinical practice.

Another more specific issue concerns our ability to generalize results collected in North American samples to clinical practice in Europe. ADHD has traditionally been recognized and managed very differently between Europe and the United States and also across the different European

\footnotetext{
'INSERM U669, Paris, France

${ }^{2}$ Univ Paris-Sud and Univ Paris Descartes, UMR-S0669, Paris, France

${ }^{3}$ AP-HP, Hôpital Paul Brousse, Département de santé publique,Villejuif,

France

${ }^{4}$ University of Dundee, Scotland, UK

${ }^{5}$ University of Goettingen, Child and Adolescent Psychiatry, Germany

${ }^{6}$ Eli Lilly and Company, Lilly Research Centre, ErI Wood Manor,

Windlesham, Surrey, UK
}

Corresponding Author:

Prof. Bruno Falisaard, INSERM U669, Maison de Solenn, 97 boulevard de Port Royal, 75679 Paris cedex I4, France

E-mail: falissard_b@wanadoo.fr 
nations (Faraone, Sergeant, Gillberg, \& Biederman, 2003). Furthermore, the range of nonpharmacological interventions used, especially psychotherapies, are likely to vary widely from country to country.

In such a cross-cultural context, it is unclear to what extent the conclusions drawn from randomized controlled trials conducted in North America can be applied to a European setting. Although observational treatment studies lack the scientific rigor of randomized controlled trials, they have different strengths. They are clearly more pragmatic and, if conducted and analyzed carefully, can not only describe current practice but also assist validating or questioning the real-world applicability of findings from the randomized controlled trials. Observational treatment studies can, therefore, either provide support for the translation of findings from U.S. clinical trials into real-world clinical settings and to non-U.S. populations or suggest the need for further more detailed population and context specific investigations of these findings.

Here we describe an analysis of data from the initial stages of the ADHD Observational Research in Europe (ADORE) study (Preuss et al., 2006). ADORE is a prospective, observational, pan-European, observational study of children with problematic inattention, impulsivity, and hyperactivity that will collect data pertaining to the first 2 years of treatment. This analysis focuses on addressing a basic clinical question: How do the early treatment choices made by the ADORE physicians when treating children with an "ADHD-related diagnosis" affect these children in the first few months following diagnosis? On the basis of data from the multimodal treatment study of ADHD (MTA Cooperative Group, 1999), which suggests that treatments plans that include a medication treatment result in more favorable outcomes in terms of ADHD symptom reduction than those plans that did not include a medication treatment, we hypothesized that these same treatment effects would be demonstrated within the early stages of treatment in a pan-European cohort of participants receiving naturalistic treatment within a real-life setting. This analysis will provide a background and context for the analysis of data from the full 2 years of the study and facilitates the comparison of early and late treatment effects in these participants. It also gives the opportunity to provide a realworld validation of the findings reported in short-term randomized controlled trials of stimulant medication.

\section{Method}

ADORE is a 2-year, longitudinal, observational, multicenter study conducted in 10 European countries, which included 1,478 participants at baseline (Preuss et al., 2006). As medical training, organization, and practice varies considerably across Europe, the physicians participating in the study came from a range of medical specialties but mainly from child psychiatry and pediatrics. All of the participating physicians were experienced in the management of ADHD, hyperactive, inattentive, impulsive symptoms and associated problems. Before the study commenced, the different profiles of physicians who diagnose and treat ADHD in each participating country were defined, and efforts were made to recruit a representative proportion of physicians from each of these medical specialties to participate in the study. All raters received inventory training at specific meetings to guarantee low variation. There was no minimum number of participants per investigator.

There were seven planned data collection points, each of which was defined by a time window. As this was an observational study, participating physicians were free to make all treatment decisions according to their usual practice, including determination of the timing of consultations. As a consequence, not all participants were seen at all time points. We describe here the data collected at the first three data collection points: Time 1 (the baseline assessment), Time 2 (the first follow-up visit, taking place in the time period between 7 days and 2 months after Time 1), and Time 3 (the first follow-up visit taking place in the time period between 2 and 5 months after Time 1). Only the first visit during each time period was recorded; for example, if a participant returned for follow-up 2 weeks after Time 1 and then again 7 weeks after Time 1, only the visit at 2 weeks would be recorded and this would be designated Time 2. As visits at each time point were not mandatory missing data for any particular visit do not imply either a violation or that a participant has dropped out of the study; it simply indicate that information is not available for that visit, as either the participant had no visit during that time period or failed to attend a scheduled appointment. In either situation, the participant remained eligible to have data collected at the next data collection time point.

The traditions and approach to child psychiatry in general, and toward ADHD in particular, varies considerably across Europe. For example, ADHD is now well accepted as a diagnosis in the United Kingdom, the Netherlands, and Germany, whereas in France and Italy, where there remains a significant influence from the psychodynamic school of thinking, ADHD is not well accepted by some clinicians. As the intention of the ADORE study was to describe the treatment of those children presenting to services across Europe with ADHD, the eligibility criteria, regardless of the diagnostic label applied, were designed to acknowledge these differences. These eligibility criterion for enrolling a child was the child must be aged between 6 and 18 years and presenting with symptoms (problems) of hyperactivity, impulsivity, or inattentiveness, as judged by the physician. A formal diagnosis of ADHD was not required, as it was felt that it would preclude many participants who were not given a diagnosis for theoretical and clinician- or culturerelated reasons rather than that they did not meet formal 
diagnostic criteria. Although this has the potential of introducing bias, it should be remembered that this was only an observational study that was seeking to describe actual practice across Europe rather than a clinical trial designed to demonstrate efficacy. The majority of participants were given a diagnosis of either hyperkinetic disorder (World Health Organisation, 1992) or ADHD (American Psychiatric Association, 1994) by the treating physician, and the ADHD rating scale scores of the participants enrolled were only slightly lower than those enrolled in typical industrysponsored clinical trials (Preuss et al., 2006). To recruit only treatment-naïve participants, participants were precluded from entering the study if they had previously been given a formal diagnosis of hyperkinetic disorder or ADHD. Other exclusion criteria included a diagnosis of autism, schizophrenia, or mental retardation or participation in another research study that included a treatment, intervention, and/or an investigational drug. Oral, informed consent (or written if required) was obtained from the participant's parent or legal guardian before enrollment. The study was approved by all of the appropriate ethical review boards.

A wide range of data were collected at each data collection point, including sociodemographic variables; ratings of school achievement; family burden; the ADHD rating scale (ADHD-RS, rated by physicians with parents as informants (DuPaul, Power, Anastopoulos, \& Reid, 1998), a measure of ADHD symptomatology; the presence of coexisting problems, as assessed by the treating physician; Children's Global Assessment of Severity (CGAS, physician completed; Shaffer et al., 1983), a measure of functional impairment; Clinical Global Impression severity scale (CGI, physician completed; Guy, 1976), a 7-point Likert scale $(1=$ normal, not ill at all to $7=$ very severely ill $)$; the Strength and Difficulties Questionnaire (SDQ, parent completed; Goodman, 1997), a brief 25-item behavioral screening questionnaire that generates 5 subscale scores (emotional symptoms, conduct problems, hyperactivity/ inattention, peer relationship problems, and prosocial behavior) and a total difficulties score; Child Health and Illness Profile-Child Edition (CHIP-CE parent version, parent completed; Riley et al., 2004), a generic measure of health-related quality of life; the CHIP-CE comprises five domains (satisfaction, comfort, resilience, risk avoidance, and achievement) and 12 subdomains. For the present analysis, we focused on parent-reported ADHD symptoms, as assessed by the physician, using the ADHD-RS.

For recording purposes, psychosocial interventions were divided into the following: psychoeducational counseling, psychodynamic therapy, cognitive behavioral therapy, family therapy, and other therapies. Number of sessions per month were documented. Prescription of speech therapy, occupational therapy, relaxation therapy, psychomotor therapy, and educational interventions within school were also recorded. Pharmacological treatments recorded were those available in Europe at the time of the study: methylphenidate, methylphenidate long-acting, other long-acting stimulants, dexamphetamine, pemoline, reboxetine, clonidine, tricyclic antidepressants (TCAs), selective serotonin reuptake inhibitors (SSRIs), antipsychotics, bupropion, and other pharmacotherapies. Doses of all pharmacological treatments were documented.

\section{Data Analysis}

The population analyzed corresponds to the 1,478 children enrolled into the study at baseline. Treatments were collapsed in two summary variables: (1) pharmacotherapy prescribed at baseline yes/no (at least one of the drugs mentioned above), and (2) psychosocial intervention prescribed at baseline yes/no (psychoeducational counseling, psychodynamic therapy, cognitive-behavioral therapy, family therapy, and other therapies). Baseline characteristics were compared across these treatment groups using the chi-square test for categorical data, using Monte-Carlo simulations when the validity of this test was not guaranteed; continuous variables were compared using the Student $t$ test. Confidence intervals (CI) were estimated from the Gaussian density for continuous variables and from a scoretest-based approach for binary variables.

The prespecified main analysis was the comparison of the change in ADHD-RS score between baseline and the last recorded visit across treatment groups. Formally, a multiple linear regression model was used, with change in ADHD-RS score as the dependent variable; the independent variables were pharmacotherapy (yes/no), psychosocial intervention (yes/no), the interaction between these two treatment variables, ADHD-RS baseline score, and three propensity scores (see below). This model was chosen over a model in which the treatment variable would have been coded through a 4-class categorical variable (pharmacotherapy, psychosocial intervention, both, none) because it is closer to the observational nature of the study. No treatment groups were defined a priori.

In real-world clinical practice a clinician does not randomly allocate participants to a particular treatment but chooses a treatment based on a variety of factors. As observational studies estimate treatment effects from these nonrandomly allocated participants, they are subject to possible "prescription biases." This prescription bias was addressed by using a propensity adjustment method (Rosenbaum \& Rubin, 1983). Propensity scores are calculated from a broad range of variables considered to be potential sources of bias in allocating individuals to particular treatments. The propensity score gives an estimate of the probability that a participant would be allocated to any particular treatment; this probability is then used to adjust the estimate of the treatment effect, thus creating a quasiexperimental design. The objective is to make treatment 
groups comparable even if there has been no randomization. Though unobserved covariates may still introduce some bias into the treatment effect estimation, this effect can be minimized when data on a large number of potentially biasing variables have been collected at baseline and are included in the calculation of the propensity score. For this analysis we computed propensity scores using logistic regression modeling of baseline variables and included 64 treatment selection covariates in the models. These included sociodemographic, clinical, and familial characteristics, or treatments not related to a medication or psychosocial intervention (full details of the covariates included in this analysis are available from the first author). Covariate interactions proved to be unnecessary. The discriminate power of the propensity scores was quantified by measurement of the receiver-operating characteristic area (the C-index), which may be considered as good if it is greater than 0.7 (Hosmer \& Lemeshow, 2001). Three propensity scores were calculated: (1) for the dependent variable: prescription of a pharmacotherapy, (2) for the prescription of a psychosocial intervention, and (3) for the prescription of both treatments together. For the computation of these propensity scores, missing data for covariates were imputed using a Gibbs sampler (van Buuren, Boshuizen, \& Knook, 1999). In all subsequent models, the three propensity scores were used as 10-class categorical variables to account for possible nonlinear relationships.

Post hoc sensitivity analyses were conducted to test the strength of the results obtained from the main analysis. They included (1) an analysis of the subsample of participant $\mathrm{s}$ who did not change treatment at Time 2 (participants with stable treatment), (2) an analysis with the addition of 23 covariates considered as potential major confounders (speech therapy, psychomotor therapy, occupational therapy, relaxation, and so on; type of physician and country; gap between Time 1 and Time 3; comorbidities [conduct disorder, oppositional defiant disorder, anxiety, depression]; ADHD-RS at baseline; CGI at baseline; quality-of-life subscores at baseline; SDQ subscores at baseline; previous treatment with a medication), (3) an analysis with all types of psychosocial interventions specified as different covariates, (4) an analysis of other clinical outcomes (CGI, CGAS, SDQ), and (5) a linear mixed model considering participants who changed treatment at Time 2 as two different participants, one for Time 1 and Time 2, another for Time 2 and Time 3.

Ideally, a thorough evaluation of psychosocial interventions would have required taking into account the number, length, and level of standard of these interventions. Unfortunately, we had no data about length, standard, and the number of sessions, which, though collected, was inadequate or incomplete and hence could not included in the models presented above. More generally, the observational nature of the study makes it difficult to determine which treatment actually received the participants when the physician decided to propose a psychological intervention. For all these reasons, we decided to change the wording of the variable "psychosocial intervention" in a more factual fashion; thus, the variable was changed to "prescribed psychosocial intervention".

All statistical analyses were conducted using the R 2.1.1 software (R Development Core Team, 2004), mixed models were estimated with the NLME package, and imputation with the MICE package. Analyses were audited by a second statistician; data management and computations were verified with the SAS 9.1 software (Statistical Analysis Software, 2004).

Type 1 error was two sided and fixed to 0.05 in all analyses. As a single primary analysis of a primary endpoint was clearly individualized, no adjustment for multiple comparisons was done.

\section{Results}

The mean age of the 1,478 children included in the analysis was 9.0 years $(S D=2.5), 84 \%$ of participants were male. Further data on the baseline characteristics of the sample have been published in a previous paper (Preuss et al., 2006).

At Time 1 (baseline), data on the treatment prescribed were available for 1,472 children. Of these, $49.9 \%$ of the children from the study sample were prescribed a medication treatment (pharmacotherapy); $43.7 \%$ of them were prescribed an immediate-release methylphenidate; $4.0 \%$ of them were prescribed a long-acting methylphenidate; and other medications provided on a relatively low scale were dexamphetamine $(0.1 \%)$, long-acting stimulants $(0.06 \%)$, and other medications (2.9\%; clonidine, antidepressants, or antipsychotics). Of those prescribed a medication at baseline, $96.1 \%$ (47.9\% of the total sample) of the participants were prescribed a stimulant medication. At baseline, 653 participants (44.3\%) were prescribed a psychosocial intervention; $33.1 \%$ were prescribed $(74.7 \%$ of those prescribed a psychosocial intervention) psychoeducational counseling; 8\% were prescribed cognitive behavioral therapy; $4.1 \%$ were prescribed family therapy; $2.8 \%$ were prescribed psychodynamic therapy; and $3.4 \%$ were prescribed another nonpharmacological therapy. The prescription of medications and psychosocial interventions at Time 1 was well balanced across the sample: 364 participants (24.7\%) were prescribed medication only; 282 (19.2\%), psychosocial intervention only; 371 (25.2\%), both medication and psychosocial intervention; and 455 (30.9\%), neither medication nor psychosocial intervention.

The baseline characteristics of participants prescribed a medication at Time 1 differed from those who were not prescribed a medication (Table 1). Participants prescribed a medication treatment were older; had higher levels of 
Table I. Comparison of Participants' Characteristics At Baseline Across Treatment Groups

\begin{tabular}{|c|c|c|c|c|c|c|}
\hline & \multicolumn{2}{|c|}{ Medication } & & \multicolumn{2}{|c|}{ Psychosocial Intervention } & \multirow[b]{3}{*}{$p$} \\
\hline & Yes $(n=735)$ & No $(n=737)$ & & Yes $(n=653)$ & No $(n=819)$ & \\
\hline & $\%(95 \% \mathrm{Cl})$ & $\%(95 \% \mathrm{Cl})$ & $p$ & $\%(95 \% \mathrm{Cl})$ & $\%(95 \% \mathrm{Cl})$ & \\
\hline Medications & & & & $57(53-60)$ & $44(4 \mid-48)$ & * \\
\hline Psychosocial interventions & $5 \mathrm{I}(47-54)$ & $38(35-42)$ & $*$ & & & * \\
\hline Other therapy & $43(40-47)$ & $43(40-47)$ & & $55(5 I-59)$ & $34(3 \mid-38)$ & $*$ \\
\hline Educational intervention in school & $26(23-30)$ & $24(2 \mid-28)$ & & $33(30-37)$ & $19(16-22)$ & * \\
\hline Speech therapy & $6(5-8)$ & $7(5-9)$ & & $9(7-12)$ & $4(3-6)$ & $*$ \\
\hline Occupational therapy & $7(5-9)$ & $7(6-10)$ & & $8(6-10)$ & $6(5-8)$ & \\
\hline Relaxation therapy & $2(1-4)$ & I $(0-2)$ & & $3(2-4)$ & $I(0-1)$ & * \\
\hline Psychomotor therapy & $4(3-6)$ & $3(2-5)$ & & $6(4-8)$ & $2(1-3)$ & $*$ \\
\hline Learning problems & $61(57-64)$ & $5 I(47-55)$ & $*$ & $60(56-64)$ & $52(49-56)$ & * \\
\hline \multirow[t]{2}{*}{ Sex (male) } & $83(80-86)$ & $85(82-87)$ & & $85(82-87)$ & $83(81-86)$ & \\
\hline & Mean $(95 \% \mathrm{Cl})$ & Mean $(95 \% \mathrm{Cl})$ & & Mean $(95 \% \mathrm{Cl})$ & Mean $(95 \% \mathrm{Cl})$ & \\
\hline Age, years & $9.3(9.1-9.5)$ & $8.7(8.5-8.8)$ & $*$ & $8.9(8.7-9.1)$ & $9.0(8.9-9.2)$ & \\
\hline ADHD-RS total & $37.1(36.5-37.8)$ & $34.5(33.9-35.2)$ & $*$ & $36.1(35.4-36.8)$ & $35.7(35.0-36.2)$ & \\
\hline ADHD-RS inattention & $19.6(19.3-19.9)$ & $18.2(17.9-18.6)$ & $*$ & $18.9(18.5-19.2)$ & $18.9(18.6-19.2)$ & \\
\hline ADHD-RS hyperactivity-impulsivity & $17.6(17.1-18.0)$ & $16.3(15.9-16.8)$ & $*$ & $17.2(16.8-17.7)$ & $16.7(16.3-17.2)$ & \\
\hline CGAS & $53.9(53.1-54.7)$ & $56.5(55.6-57.4)$ & * & $53.4(52.6-54.3)$ & $56.7(55.9-57.5)$ & * \\
\hline CGI & $4.5(4.4-4.6)$ & $4.2(4.2-4.3)$ & $*$ & $4.5(4.4-4.6)$ & $4.3(4.2-4.3)$ & * \\
\hline CHIP satisfaction & $31.4(30.4-32.5)$ & $34.2(33.2-35.2)$ & $*$ & $32.3(31.2-33.4)$ & $33.2(32.2-34.1)$ & \\
\hline CHIP comfort & $4 I .8(41.0-42.6)$ & $43.2(42.5-43.9)$ & $*$ & $42.2(41.4-43.0)$ & $42.7(4 \mid .9-43.4)$ & \\
\hline CHIP resilience & $35.0(34.1-35.9)$ & $37.2(36.3-38.0)$ & $*$ & $34.7(33.8-35.6)$ & $37.1(36.3-38.0)$ & * \\
\hline CHIP risk avoidance & $28.1(27.1-29.1)$ & $31.8(30.9-32.7)$ & $*$ & $28.6(27.5-29.6)$ & $31.1(30.1-32.0)$ & * \\
\hline CHIP achievement & $28.8(28.0-29.6)$ & $31.8(31.0-32.5)$ & * & $29.6(28.7-30.4)$ & $30.8(30.1-3 \mid .6)$ & * \\
\hline Academic performance & $30.6(29.8-31.4)$ & $33.2(32.4-33.9)$ & $*$ & $31.9(31.2-32.7)$ & $31.8(3||-32.6)$. & \\
\hline Relations with peers & 34.7 (33.7-35.7) & $36.7(35.8-37.7)$ & $*$ & $34.2(33.1-35.3)$ & $36.9(36.0-37.8)$ & * \\
\hline SDQ emotion & $4.2(4.0-4.4)$ & $3.7(3.6-3.9)$ & * & $4.0(3.8-4.2)$ & $3.9(3.7-4.1)$ & \\
\hline SDQ conduct problems & $4.7(4.5-4.9)$ & $4.2(4.1-4.4)$ & $*$ & $4.7(4.5-4.9)$ & $4.3(4.1-4.5)$ & * \\
\hline SDQ hyperactivity & $8.4(8.2-8.5)$ & $8.2(8.1-8.3)$ & * & $8.4(8.2-8.5)$ & $8.2(8.1-8.3)$ & \\
\hline SDQ relations with peers & $3.8(3.6-4.0)$ & $3.5(3.3-3.7)$ & * & $4.0(3.8-4.2)$ & $3.4(3.3-3.6)$ & $*$ \\
\hline SDQ prosocial behaviors & $6.7(6.5-6.9)$ & $7.0(6.8-7.1)$ & $*$ & $6.8(6.6-6.9)$ & $6.9(6.7-7.0)$ & \\
\hline SDQ total & $21.1(20.6-2 \mid .5)$ & $19.6(19.2-20.1)$ & $*$ & $21.0(20.6-21.5)$ & $19.8(19.4-20.2)$ & * \\
\hline Years since treatment sought & $1.9(1.7-2.0)$ & $1.6(1.4-1.7)$ & $*$ & $1.7(1.5-1.9)$ & $1.7(1.6-1.9)$ & \\
\hline $\begin{array}{l}\text { Time between Time I and Time } \\
3 \text { (days) }\end{array}$ & 95.9 & 86.4 & $*$ & 93.0 & 89.7 & \\
\hline
\end{tabular}

Note: Data on treatments prescribed at Time I were available for I,472 participants.

${ }^{*} p<0.05$ for medication versus no medication or for psychosocial intervention versus no psychosocial intervention (using chi-square tests for categorical data and Students $t$ tests for continuous data).

ADHD symptoms; higher levels of emotional, conduct, relationship, and socialization problems as measured by the SDQ; increased impairment on the CGAS and CGI; poorer academic achievement; poorer quality of life on the CHIP$\mathrm{CE}$; and were more likely to have learning problems and to receive psychosocial intervention. Differences in baseline characteristics were also observed between participants who were and were not prescribed a psychosocial intervention at Time 1. Participants prescribed a psychosocial intervention had increased impairment on CGAS and CGI; poorer resilience, risk avoidance, and achievement on the CHIP-CE; higher levels of conduct problems and relations with peers on the SDQ; were more likely to receive medications, other therapy, educational interventions at school, speech therapy, relaxation therapy, and psychomotor therapy; and were more likely to have learning problems.

Of the 1,478 participants in the analysis, data on treatments prescribed were available for 1,472 participants (99\%) at Time 1, for 1,164 participants (79\%) at Time 2, and for 1,112 participants (75\%) at Time 3. Treatments prescribed changed over time in a complex manner (Figure 1). As anticipated, the frequency of participants' prescribed medication only or medication plus psychosocial intervention was fairly consistent over time. However, $21 \%$ of the participants prescribed psychosocial intervention only at Time 1 were prescribed medication at Time 2, and most of the participants not seen at Time 2 or Time 3 had no medications prescribed at Time 1 . 


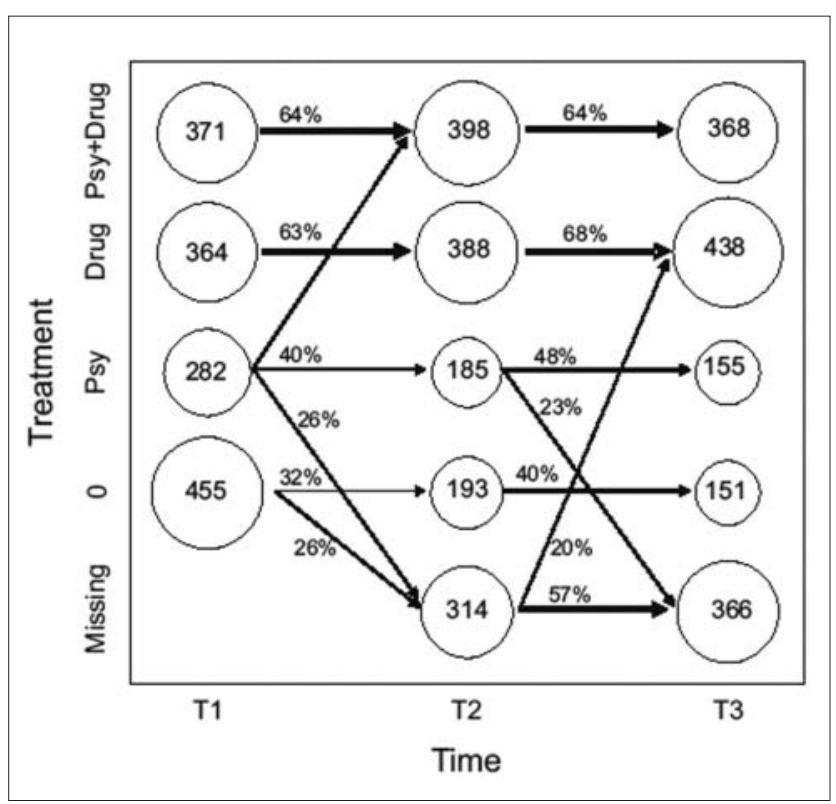

Figure I. Change in treatments prescribed across time Note: The circles give the number of participants prescribed that treatment (medication, psychosocial intervention, both medication and psychosocial intervention, neither medication nor psychosocial intervention) at times Time I (baseline), Time 2 (I week to 2 months) and Time 3 (2 to 5 months). The arrows show the percentage of participants maintaining or changing treatment between time points (arrows $<20 \%$ are not presented).

The propensity scores for medication (PSM), prescribed psychosocial intervention (PSP), and the combination of both treatments (PSMP) had a C index of 0.70, 0.73, and 0.73 , respectively. This suggests that the propensity scores can be effective in managing the bias induced by the absence of randomization of treatment at baseline (Rosenbaum \& Rubin, 1983).

Figure 2 shows the evolution of the ADHD-RS score over time for the different treatment groups (as defined at Time 1). Though all groups showed an improvement (reduction) of their ADHD symptoms at both Time 2 and Time 3, the two groups taking a medication had a greater reduction in ADHD-RS. These differences were already apparent by Time 2 .

The effects of treatment (as defined at Time 1) on the ADHD-RS scores are presented in Table 2. For the primary analysis in all participants the effect of treatments prescribed at Time 1 on the difference in the ADHD-RS score between last recorded visit and Time 1 was investigated. Compared to no treatment, there was a positive effect of medications, a negative effect of prescribed psychosocial interventions, and a nonsignificant interaction between medications and prescribed psychosocial interventions.

When the analyses were repeated for each type of prescribed psychosocial intervention (psychoeducational counseling, yes/no; cognitive-behavioral therapy, yes/no; family therapy, yes/no; psychodynamic therapy, yes/no;

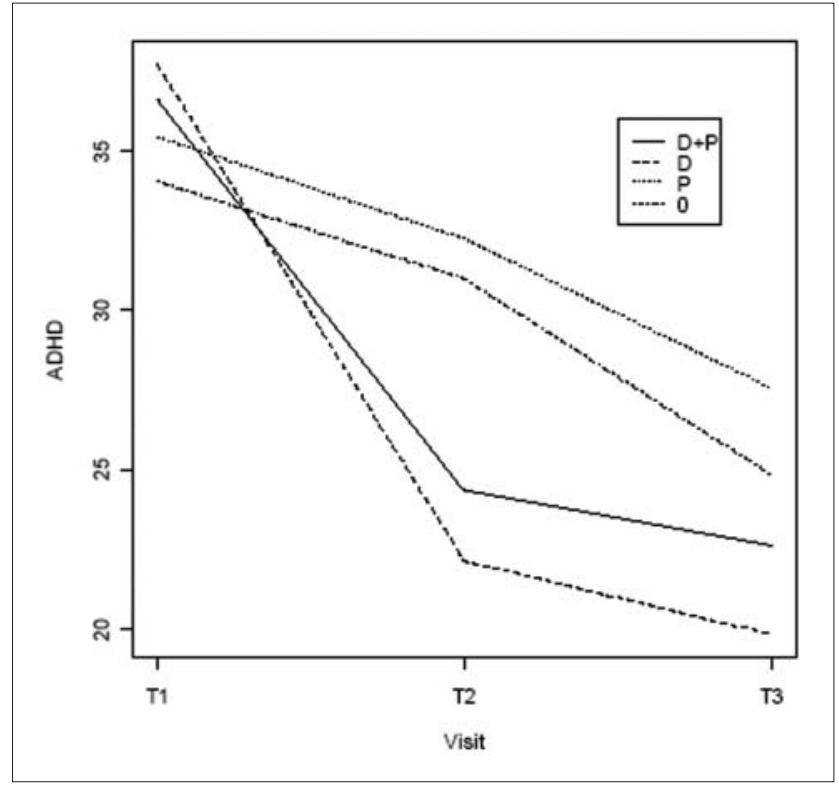

Figure 2. Unadjusted mean ADHD-RS scores at Time I, Time 2 and Time 3 for participants prescribed medication only $(D)$, psychosocial intervention only $(P)$, both medication plus psychosocial intervention $(D+P)$ or neither medication nor psychosocial intervention ( 0$)$ at baseline.

Note: A higher score indicates a worse symptom severity.

and another psychosocial intervention, yes/no), these last variables were not associated with the outcome except for psychoeducational counseling. It is, however, important to note that the groups included in these analyses were considerably smaller than those in the previous analyses and that this will have substantially decreased the statistical power. Even so, it was the case that for each type of prescribed psychosocial interventions the sign of the coefficients corresponded to a negative effect of treatment on outcome (data not shown).

The results were similar when the CGI, CGAS, or total score of the SDQ was used as the dependent variable, following the introduction of the major prognostic factors as covariates in the model, alongside the propensity scores, and when the interaction term was dropped. In each case, the prescription of medication still resulted in a significant positive effect on outcome while the prescription of prescribed psychosocial interventions resulted in a significant negative effect on outcome (data not shown). A regression analysis was performed successfully demonstrating that the assumptions required by the linear regression modelwere fulfilled.

The model that includes the clinical global impression as dependent variable can help interpret the clinical relevance of the symptom-level changes across groups. There is about 0.53 points of difference between the medication and the nonmedication groups (adjusted mean) and about 0.18 
Table 2. Effect of Pharmacotherapy (Med), Psychosocial Intervention (Psy) and Interaction (Med $\times$ Psy) on the Variations in the ADHD-RS Scores

\begin{tabular}{|c|c|c|c|}
\hline Independent variable & $\begin{array}{l}\text { Regression } \\
\text { Coefficient }\end{array}$ & $\begin{array}{c}\text { Standard } \\
\text { Error }\end{array}$ & $p$-Value \\
\hline \multicolumn{4}{|l|}{ All participants } \\
\hline Med & -3.12 & 0.29 & $<.0001$ \\
\hline Psy & 1.03 & 0.30 & .0006 \\
\hline Med $\times$ Psy & 0.35 & 0.28 & .15 \\
\hline ADHD-RS Time I & -0.40 & 0.03 & $<.0001$ \\
\hline PSM & & & NS \\
\hline PSP & & & NS \\
\hline PSMP & & & NS \\
\hline \multicolumn{4}{|l|}{$\begin{array}{l}\text { Participants with stable } \\
\text { treatment }\end{array}$} \\
\hline Med & -4.48 & 0.32 & $<.0001$ \\
\hline Psy & 0.45 & 0.34 & .18 \\
\hline Med $\times$ Psy & 0.96 & 0.31 & .002 \\
\hline ADHD-RS Time I & -0.34 & 0.04 & $<.0001$ \\
\hline PSM & & & NS \\
\hline PSP & & & NS \\
\hline PSMP & & & NS \\
\hline \multicolumn{4}{|l|}{ Mixed model } \\
\hline Med & -4.56 & 0.26 & $<.0001$ \\
\hline Psy & -0.03 & 0.26 & .91 \\
\hline Med $\times$ Psy & 0.53 & 0.25 & .032 \\
\hline ADHD-RS Time I & -0.49 & 0.02 & $<.0001$ \\
\hline PSM & & & NS \\
\hline PSP & & & NS \\
\hline PSMP & & & NS \\
\hline
\end{tabular}

Note: PSM, PSP, and PSMP denote the propensity scores respectively for medication/no medication, psychosocial intervention/no psychosocial intervention and both/no both. Since these propensity scores are categorical variables with 10 classes, coefficients are not presented.

between the prescribed psychosocial intervention and nonprescribed psychosocial intervention groups.

Table 2 also shows that in the subsample of participants with stable treatment the negative effect of prescribed psychosocial interventions became nonsignificant $(p=.18)$, but the interaction pharmacotherapy by prescribed psychosocial intervention was positive and significant $(p=.002)$. As a negative coefficient corresponds to an improvement in symptoms, this suggests that the addition of prescribed psychosocial intervention to a pharmacotherapy worsened the symptomatology, compared to the group with pharmacotherapy alone. With the mixed model, the effect of prescribed psychosocial interventions was close to 0 and nonsignificant ( $p=.91$ ), and the interaction pharmacotherapy by prescribed psychosocial intervention was positive and significant $(p=$ .032), which leads to a similar conclusion.

As the ADORE study is in a pan-European cohort, it is of interest to look for a potential difference in treatment effectiveness across countries. For this, three more factors were introduced in the model: country, medication by country, and prescribed psychosocial intervention by country.
Only the prescribed psychosocial intervention by country interaction term was statistically significant $(p<.0001)$, suggesting that prescribed psychosocial interventions had a different level of effectiveness across countries, with the highest levels of effectiveness in the Netherlands, Norway, and Austria, and the lowest levels of effectiveness in Iceland, Denmark, and the United Kingdom.

\section{Discussion}

There is now considerable evidence from randomly controlled clinical trials to support the efficacy and effectiveness of a range of pharmacological, and to some extent, nonpharmacological treatments for ADHD. Systematic reviews of this evidence consistently support these findings and their routine use in treating ADHD (American Academy of Pediatrics, 2001; Banaschewski et al., 2006; Jadad, Boyle, Cunningham, Kim, \& Schachar, 2000; King et al., 2006; Klassen, Miller, Raina, Lee, \& Olsen, 1999; Scottish Intercollegiate Guidelines Network, 2001). Pharmacological treatments for ADHD have been demonstrated to be more powerful than nonpharmacological treatments in terms of effect size; however, the most powerful treatment is not always the most appropriate (Santosh \& Taylor, 2000). There are longstanding differences in the traditional approach to diagnosing and managing ADHD between clinicians in the United States and those in Europe. The more restrictive ICD-10 diagnostic (World Health Organisation, 1992) criteria have traditionally been preferred in Europe. Hyperkinetic disorder (ICD-10) describes a subset of those children with ADHD combined type (DSM-IV; American Psychiatric Association, 1994) who are suffering from the most pervasive and disabling form of this disorder. In the United States, pharmacotherapy has for some considerable time been the first-line treatment for most participants meeting the broader DSM-IV ADHD diagnosis. In contrast, medication in Europe has traditionally been reserved for those with the more restrictive hyperkinetic disorder diagnosis. Recent years have seen some shift in this position, and current European guidelines recommend that medication is often the appropriate initial treatment for participants with severe, pervasively impairing ADHD, whereas those with less impairing or situation-specific symptoms (broader phenotype ADHD) should be offered medication as a treatment option if they fail to respond to an initial trial of psychosocial treatment (Taylor et al., 2004). In ADORE, we found that children prescribed medication at the baseline visit had more severe ADHD symptoms and impairment compared with those not prescribed medication at baseline.

A reanalysis of the influential MTA study dataset also supported the use of medication in participants with more severe ADHD (Santosh et al., 2005). The original MTA analysis suggested that a carefully crafted and executed 
medication treatment protocol was a more effective treatment for children with ADHD combined type than either regular community care or an intensive package of psychosocial treatment and that the addition of the psychosocial treatment to the medication protocol added little further benefit (MTA Cooperative Group, 1999). In their reanalysis of this dataset, Santosh and colleagues (2005) identified the subgroup of children who met the criteria for ICD-10 hyperkinetic disorder (approximately 25\% of the original sample) and compared the impact of treatment on this group compared to those with the broader ADHD phenotype. They concluded that for the group with hyperkinetic disorder, the medication and combined treatments remained the most potent and effective treatments. However, for the group with ADHD combined type who did not meet criteria for hyperkinetic disorder, there was little difference between the medication and behavioral treatments. Thus, it is to be expected that clinicians in Europe will continue to use a broader range of treatments than in the United States, depending on severity of the disorder and availability of treatment options. Despite seeming support for the European Guidelines position, there has not previously been a formal investigation of either European physician' management of ADHD in actual clinical practice or any evidence of the effectiveness of clinic-based treatments for ADHD across Europe within real-life observational settings as opposed to a experimental setting (randomized controlled trials with standardized treatments). The data reported here provide a starting point for such investigation by describing practice across a wide range of countries and settings and initial data on the effectiveness of differing approaches to treatment.

The results presented for a short-term treatment situation provide support for many of the arguments discussed above. They are generally in favor of the prescription of a medication treatment over either psychosocial intervention or no treatment. The prescription of psychosocial intervention provided no benefit over medication alone and was actually associated with a minor increase in ADHD symptoms in the short term, although this last finding requires further verification before practical decisions can be inferred and clear recommendations made, especially because nothing is known about which intervention the participant did receive in practice. It is, however, possible that the very beginning of the management of a child with ADHD-like problems is not the best time to start a psychosocial intervention. Waiting a few months until medication has improved symptoms may make the child/parents more open to invest, for example, in a psychotherapeutic process.

We have conducted a broad range of statistical analyses to take into account the observational nature of the study and consequent lack of randomization to treatment. While there are certain discrepancies between results (e.g., in the main analysis psychosocial intervention had a negative effect, and in most of the secondary analyses this factor had no effect on its own; however, the addition of a psychosocial intervention to medication had a negative effect), they are convergent in that psychosocial interventions do not appear to be associated with a positive outcome at this point in the child's treatment.

These results need to be seen in context, and interpretations have to be considered with extreme care, along with taking into account the study's limitations. This was an observational study that included participants not only from many countries but also from many centers within those countries $(n=245)$. Most physicians were in routine clinical practice with little research experience; thus, while all raters receive training on how to use the assessment and measurement techniques employed during the study at country-specific rater training meetings, similar to those used to train raters in clinical trials, some variation in practice and accuracy is to be expected. As discussed earlier, our study is observational only, and its goal was to only observe and describe practice, including any similarities and differences between countries, cultures, and so on, across Europe. In view of different practices and attitudes to ADHD across various countries and in order to facilitate generalization of the results, the participants were recruited based on the clinicians' assessment of potential participants who were presenting with symptoms such as inattention, hyperactivity, impulsivity problems, or symptoms rather than conducting a a formal diagnosis to determine ADHD or hyperkinetic disorder. This approach also represents the only way to tackle the question of effectiveness by studying the effect of a treatment on participants who actually receive it rather than those who "should" receive it. It should be noted, however, the majority (93\%) of participants did in fact receive a diagnosis of ADHD or hyperkinetic disorder: Many physicians (43\%) used DSM-IV criteria only, 32\% used ICD-10 criteria only, and 12\% used both diagnostic systems (Preuss et al., 2006). Interestingly, the ADORE sample as a whole was rated as only slightly less symptomatic and impaired than the typical samples included in randomized, controlled, multicentre medication trials (Preuss et al., 2006).

Additional limitations inherent to the observational design include the absence of randomization and blinding in the administration and outcome evaluation of treatments. Thus, even if measurements are robust these represent a source of potential bias. In addition, even though a large number of potential confounders were taken into account within the propensity analysis, there is always the possibility of an imbalance in one or several unmeasured confounder(s). As is common in clinical trials, participants who attended all visits had different characteristics than those who didn't. However, the analyses using a lastobservation-carried-forward procedure (LOCF) gave results similar to the analyses on the full attenders and on the population of participants with stable treatments. 
Concerning the negative effect of psychosocial interventions, we need to be particularly cautious in our interpretations. First, in the primary analysis, the actual difference of about 2 points in ADHD-RS scores, following treatment between the two groups (psychosocial intervention prescribed at baseline, yes/no), is unlikely to be clinically relevant (it corresponds to a difference of less than 0.2 points in the CGI when CGI was used as the dependent variable in the model). Second, we combined the various types of psychosocial intervention into a single group and may, therefore, have diluted the effectiveness of any one specific type of psychosocial intervention. Third, we do not know whether any participant actually received his or her sessions of psychosocial intervention or whether this therapy was carried out according to acceptable standards. As mentioned in the Methods section, the variable studied here is, "The physician has indicated, at Time 1, that they are prescribing a psychological treatment" and not that "The patient has received a psychological treatment following recognized practices." Most of the "psychosocial interventions" given were described as "psychoeducation and counseling" rather than as a specific program of a recognized "psychotherapy" (e.g., behavioral parent training) that has been previously demonstrated to be efficacious in managing ADHD. Lastly, we have here only considered the short-term perspective, and it is likely that many psychosocial interventions require more time to generate a positive effect. This may become clearer when the longer-term data from the study are analyzed.

Notwithstanding these limitations, there are several strengths to the study data. They describe the impact of early treatment on participants with ADHD who are new to treatment in real-world clinical settings across Europe. Treatments are evaluated here according to what is actually done in day-to-day practice and not according to what should be done in an optimal circumstance. In contrast to the highly selected participant groups seen in clinical trials, the participants in the ADORE study are more representative of those participants actually referred for treatment. The results are consistent with expectations drawn from the literature and provide a degree of external validity to these findings. The clinicians appear to be following the recommendations of the latest European Guidelines (Taylor et al., 2004) by prescribing medication as a first-line treatment for those with more severe symptoms and psychosocial intervention for an intermediate group, and, where it was decided to defer treatment decisions, it is for those participants with lesser degrees of difficulty. More important, the results are consistent across a wide range of outcome measures that cover not only ADHD symptomatology but also clinician-rated global impairment and parent ratings of overall psychopathology.

\section{Conclusion}

Taken together, these findings support the early effectiveness of medications in reducing ADHD symptoms and impairments in routine clinical practice in Europe. A variety of psychosocial interventions are being offered in the early stages to many participants, but there is little homogeneity, and, when they are grouped together for analysis, they seem to have little positive impact. This last results, however, have to be interpreted cautiously because nothing is known about which psychosocial intervention the participant actually received in practice and even if he or she received it at all.

\section{Acknowledgments}

The study sponsor (Eli Lilly and Company) supplied the data to the group performing the analysis, but did not give any funding support for this work or influence the content of this manuscript in any way. The statistical analyses for the manuscript were independently performed by Bruno Falissard. The authors thank Deirdre Elmhirst, $\mathrm{PhD}$, for helping to edit the manuscript.

\section{Disclosure}

The authors were free to analyze and write the paper. BF has received fees for consultancies or conferences from SanofiAventis, Pierre Fabre, Lilly, Janssen, BMS, Lundbeck, Servier, GSK; DC has received fees for consultancies or conferences from Eli Lilly, Janssen Cilag, UCB Pharma, Shire, Medice, Cephalon, Pfizer. AR has received fees for consultations and conferences from Medice, Lilly, Janssen-Cilag, Shire, UCB, Cephalon

\section{References}

Abikoff, H., McGough, J., Vitiello, B., McCracken, J., Davies, M., Walkup, J., et al. (2005). Sequential pharmacotherapy for children with comorbid attention-deficit/hyperactivity and anxiety disorders. Journal of the American Academy of Child and Adolescent Psychiatry, 44, 418-427.

American Academy of Pediatrics. (2001). Clinical practice guideline: Treatment of the school-aged child with attention-deficit/ hyperactivity disorder. Pediatrics, 108, 1033-1044.

American Psychiatric Association. (1994). DSM-IV. Diagnostic and statistical manual of mental disorders (4th ed.). Washington, DC: Author.

Banaschewski, T., Coghill, D., Santosh, P., Zuddas, A., Asherson, P., Buitelaar, J., et al. (2006). Long-acting medications for the hyperkinetic disorders. A systematic review and European treatment guideline. European Child \& Adolescent Psychiatry, 15, 476-495.

DuPaul, G., Power, T., Anastopoulos, A., \& Reid, R. (1998). ADHD Rating Scale-IV: Checklists, norms, and clinical interpretation. New York: Guilford.

Faraone, S. V., Sergeant, J., Gillberg, C., \& Biederman, J. (2003). The worldwide prevalence of ADHD: Is it an American condition? World Psychiatry, 2, 104-113. 
Goodman, R. (1997). The Strengths and Difficulties Questionnaire: A research note. Journal of Child Psychology and Psychiatry, and Allied Disciplines, 38, 581-586.

Guy, W. (1976). Clinical Global Impression. ECDEU. Assessment manual for psychopharmacology-revised. Rockville, MD: National Institute of Mental Health.

Hosmer, D., \& Lemeshow, S. (2001). Applied logistic regression (2nd ed.). New York: John Wiley.

Jadad, A., Boyle, M., Cunningham, C., Kim, M., \& Schachar, R. (2000). Treatment of attention-deficit/hyperactivity disorder. Evidence Report: Technology Assessment (Summary), 11, i-viii.

King, S., Griffin, S., Hodges, Z., Weatherly, H., Asseburg, C., Richardson, G., et al. (2006). A systematic review and economic model of the effectiveness and cost-effectiveness of methylphenidate, dexamfetamine and atomoxetine for the treatment of attention deficit hyperactivity disorder in children and adolescents. Health Technology Assessment, 10, iii-iv, xiii-146.

Klassen, A., Miller, A., Raina, P., Lee, S. K., \& Olsen, L. (1999). Attention-deficit hyperactivity disorder in children and youth: A quantitative systematic review of the efficacy of different management strategies. Canadian Journal of Psychiatry, 44, 1007-1016.

McClellan, J. M., \& Werry, J. S. (2003). Evidence-based treatments in child and adolescent psychiatry: An inventory. Journal of the American Academy of Child and Adolescent Psychiatry, 42, 1388-1400.

MTA Cooperative Group. (1999). A 14-month randomized clinical trial of treatment strategies for attention-deficit/hyperactivity disorder. The MTA Cooperative Group. Multimodal treatment study of children with ADHD. Archives of General Psychiatry, 56, 1073-1086.

Preuss, U., Ralston, S. J., Baldursson, G., Falissard, B., Lorenzo, M. J., Rodrigues Pereira, R., et al. (2006). Study design, baseline patient characteristics and intervention in a cross-cultural framework: Results from the ADORE study. European Child \& Adolescent Psychiatry, 15(Suppl. 1), i4-i14.

R Development Core Team. (2004). R: A language and environment for statistical computing. Available from http://www.Rproject.org

Rappley, M. D. (2005). Clinical practice. Attention deficithyperactivity disorder. New England Journal of Medicine, 352, 165-173.

Riley, A. W., Forrest, C. B., Starfield, B., Rebok, G. W., Robertson, J. A., \& Green, B. F. (2004). The parent report form of the CHIP-child edition: Reliability and validity. Medical Care, 42, 210-220.

Rosenbaum, P., \& Rubin, D. (1983). The central role of the propensity score in observational studies for causal effect. Biometrika, 70, 41-55.

Santosh, P., \& Taylor, E. (2000). Stimulant drugs. European Child and Adolescent Psychiatry, 9 (Suppl. 1), 127-143.
Santosh, P., Taylor, E., Swansonc, J., Wigalc, T., Chuangd, S., Daviese, M., et al. (2005). Refining the diagnoses of inattention and overactivity syndromes: A reanalysis of the Multimodal Treatment study of attention deficit hyperactivity disorder (ADHD) based on ICD-10 criteria for hyperkinetic disorder. Clinical Neuroscience Research, 5, 307-314.

Scottish Intercollegiate Guidelines Network. (2001). Attention deficit and hyperkinetic disorders in children and young people: A national clinical guideline (SIGN Publication No. 52). Edinburgh: Author.

Shaffer, D., Gould, M. S., Brasic, J., Ambrosini, P., Fisher, P., Bird, H., et al. (1983). A children's global assessment scale (CGAS). Archives of General Psychiatry, 40, 1228-1231.

Statistical Analysis Software. (2004). 9.1 ed. Cary, NC: SAS Institute.

Strom, B. L. (2005). Pharmacoepidemiology (4th ed.). Chichester, UK: Wiley.

Taylor, E., Dopfner, M., Sergeant, J., Asherson, P., Banaschewski, T., Buitelaar, J., et al. (2004). European clinical guidelines for hyperkinetic disorder-First upgrade. European Child \& Adolescent Psychiatry, 13(Suppl. 1), I7-30.

van Buuren, S., Boshuizen, H. C., \& Knook, D. L. (1999). Multiple imputation of missing blood pressure covariates in survival analysis. Statistics in Medicine, 18, 681-694.

World Health Organisation. (1992). International Statistical Classification of Diseases and Related Health Problems, Tenth Revision. Geneva: Author.

\section{Bios}

Prof. Dr. Aribert Rothenberger is Chair and Director of the Department of Child and Adolescent Psychiatry at the University of Goettingen, Germany. His main clinical and research interest is in neuropsychiatry of tic disorders and ADHD, with his cornerstone study being the relationship between brain electrical activity and behavior.

Maria Lorenzo, MSc, European Health Outcomes, is a full time employee of Eli Lilly and Company. She is responsible for leading and coordinating the ADORE study activities.

Dr. David Coghill leads the Developmental Research Group at the University of Dundee. His group's research interests are focused on ADHD and other developmental disorders and include studies into the neuropsychology of ADHD and conduct disorder, neuropsychopharmacoloogy, genetics, pharmacogenetics, clinical trials, quality of life, and translational studies investigating the best ways to integrate scientific knowledge into clinical practice.

Prof. Bruno Falissard is child and adolescent psychiatrist and professor in biostatistics at the South-Paris University. He leads the INSERM unit 669 dedicated to public health in mental health. 